\title{
Reputational Concerns in Arbitration: Decision Bias and Information Acquisition*
}

\author{
Elisabetta Iossa ${ }^{\dagger}$
}

June 2007

\begin{abstract}
We analyze how reputational concerns of arbitrators affect the quality of their decision process, in particular, information acquisition and bias. We assume that arbitrators differ in their ability to observe the state of the world and that information acquisition is costly and unobservable. We show that reputational concerns increase incentives for information acquisition but may induce arbitrators to bias their decisions towards one party in the dispute. This decision bias is greater when the dispute proceedings are confidential rather than public.

Building on these results, we study the circumstances under which the parties to a contract choose to employ arbitration rather than litigation in court to resolve their disputes.
\end{abstract}

Keywords: arbitration, confidentiality, decision making, experts, information acquisition, reputational concerns.

JEL Classification: D83, K41, J52

\section{Introduction}

In this paper we study how reputational concerns of decision makers impact on the quality of their decision process, in particular information acquisition and bias, and how this affects the desirability of non-judicial institutions for dispute resolution. We focus on the case of arbitration in commercial transactions.

\footnotetext{
${ }^{*}$ For helpful comments or discussions, I wish to thank Sylvain Bourjade, Erik Eyster, Leonardo Felli, Julian Greenhill, Paul Grout, Bruno Jullien, David Martimort, Patrick Rey, Neil Rickman, Jean-Charles Rochet, Alan Schwartz, Giancarlo Spagnolo, seminar participants at Brunel University, IDEI Toulouse, University of Bath, University of Surrey, University of Tor Vergata and University of Bristol, and conference participants at Public Economics UK Autumn Conference, Oxford. Special thanks go to Giuliana Palumbo for our long discussions on the topic. An earlier version of this paper was circulated under the title "Reputational Concerns and Bias in Arbitration".

${ }^{\dagger}$ Economics and Finance Section and CEDI, School of Social Sciences, Brunel University; DEI, University of Rome Tor Vergata; and CMPO University of Bristol. Part of this paper was written whilst this author was visiting IDEI, University of Toulouse, which is thanked for the hospitality.
} 
During contract negotiations, the parties to the contract may agree to insert a clause (the 'arbitration clause') providing for the use of arbitration as dispute-resolution mechanism. ${ }^{1}$ In the event that a dispute arises, the parties then submit the dispute to an arbitrator (or to an arbitral tribunal) who conducts the arbitral hearing and issues an award that binds the parties. The arbitration clause usually defines the process by which the arbitrator will be selected; often it also specifies the qualifications the arbitrator is required to have or his/her identity.

There are different selection procedures. For example, parties may consult, or eliminate and rank, names from lists of arbitrators maintained by arbitration institutions such as the American Arbitration Association (AAA). Parties may also opt for a tripartite arbitral panel, where each party appoints her own arbitrator and then the party-appointed arbitrators select a third arbitrator, who serves as the chair. A common aspect of the procedures is that parties' preferences are taken into account in the selection of the arbitrator(s), making arbitrators' future earning prospects dependent on them being in demand. The reputational concerns of arbitrators towards the parties is the first main feature of arbitration, parties preferences playing no role in the selection of court judges.

The second main feature of arbitration is finality. Under the court system, lower-court decisions can be appealed on questions of law or (in some instances) of fact, and then be reversed by an appeals court. Instead, most arbitral proceedings purport to be final and binding, with the result that courts are reluctant to review arbitral awards, even if it is alleged that the arbitrator found facts unsupported by evidence or misapplied the law. ${ }^{2}$

The third main feature of arbitration is confidentiality. Arbitration is not a public proceeding, contrary to trial before court. The arbitration clause may contain a confidentiality provision establishing that contractual disputes will be kept confidential to the parties. ${ }^{3}$

In this paper we analyze the decision of the parties to choose arbitration rather than litigation in court. We focus on how reputational concerns affect the arbitrator's decision and his effort, but we also study the role played by lack of appeals and confidentiality provision. We show that arbitration works best when both parties in the dispute are corporations, as

\footnotetext{
${ }^{1}$ We focus on 'ex ante' arbitration agreements as opposed to 'ex post' arbitration agreements where the parties agree to employ arbitration once a dispute has already arisen.

${ }^{2}$ One of the few grounds for vacating an arbitral award is that the arbitrator was corrupt, exceeded his powers or there was evidence of partiality.

${ }^{3}$ Confidentiality is also the default option under many arbitration rules, such as the rules of the the London Court of International Arbitration (LCIA).
} 
reputational concerns generate benefits and no cost when both parties in the dispute are long-lived. Disputes among individuals or between individuals and corporations are better solved through litigation in court. Arbitration is also most beneficial for issues that are not overly complex or when the value of confidentiality for the parties is high. Efficient appeals systems instead call for litigation in court.

We incorporate the three main features of arbitration in a stylized model where arbitrators have private information about their competency. More competent arbitrators are able to acquire better information on the state of the world, i.e. the correct decision resolving a dispute. The parties to the dispute have some private information about the state of the world and have optimistic priors. Arbitrators care about appearing competent to the outside market and to any party in the dispute who may need their service in the future. We refer to such parties as 'long-lived' parties. 'Short-lived' parties are those unlikely to need arbitral services again, say because of the limited extent of their business activities.

In this setting, reputational concerns with the parties generate two contrasting effects. On the one hand, they increase the incentive for competent arbitrators to acquire costly and unverifiable information on the state of the world. On the other hand, reputational concerns induce decision bias by incompetent and uninformed arbitrators when a party in the dispute is long-lived whilst the other one is short-lived.

The incentive of a competent arbitrator to acquire information comes from the parties being more inclined to re-hire arbitrators who take correct decisions. Taking the correct decision is more likely when the arbitrator is informed; thus information helps to signal competency and to enhance future earning prospects.

The incentive of an incompetent and uninformed arbitrator to bias his decision comes from the parties having biased priors. Ceteris paribus, a party is more likely to believe that a decision is correct if the outcome is in her interest. When only one party in the dispute is long-lived, the incompetent arbitrator will then bias his decision in favour of this party in order to appear competent to her and enhance future earning prospects.

We further show that confidentiality provision increases decision bias. This is because the market anticipates the direction of the bias and updates its belief about the competency of arbitrator accordingly. As it is less competent arbitrators who bias their decisions, market updating makes decision bias less rewarding. Confidentiality also reduces the incentives of 
the arbitrator to acquire information, because of its effect on the decision bias. Predictions are then derived regarding the choice of confidentiality. When the parties are asymmetric and they expect technical issues to arise in the event of a dispute, they will opt for no confidentiality provision. Intuitively, when the subject matter is complex, providing incentives to acquire information is critical. To increase incentives, the parties to the dispute may give up confidentiality. Instead, confidentiality brings no cost to symmetric parties who are therefore likely to always choose confidentiality.

Our results suggest that arbitrators have an incentive to favour long-lived parties, such as corporations, at the expense of short-lived parties, such as individuals or small businesses. In fact, because of the efficiency cost of decision bias, asymmetric parties are less likely to agree to employ arbitration as a dispute-resolution process than symmetric parties. Symmetric and long-lived parties are the most likely to choose arbitration; they face no decision bias and strongest incentives for arbitrators to acquire information.

In the final part of the paper we formalize this intuition by explicitly comparing arbitration with litigation in court, assuming that under the latter the decision maker has no reputational concerns with the parties, the dispute proceedings are public and appeals are allowed. Our results highlight the difference in incentives to decision makers under arbitration and under litigation in court as one between ex ante and ex-post monitoring. Ex-ante monitoring is the selection of arbitrators by the parties, whilst ex-post monitoring is the appeals system. ${ }^{4}$

Arbitrator bias is not uncommon. In the seminal U.S. Supreme Court Case Commonwealth Coating Cort. v. Continental Casualty Co. (395 U.S. 145, 1968) one of the parties discovered that the neutral arbitrator had previously provided certain consulting services for the other party, and failure to disclose the commercial relationship was viewed as a manifest violation of his duties. Since Commonwealth Coatings, a number of courts have considered repeated economic contacts of one form or another as relevant evidence on the question of arbitrator bias. Bloom and Cavanagh (1986) give evidence of arbitrator bias in favour of the employer in wage disputes in labour relationships. Repeated players bias is also found and discussed in Bingham (1998).

The rest of the paper is organized as follows. In section 2 we discuss the related literature, whilst in section 3 we set up the model. Section 4 analyzes the equilibrium when the award is confidential whilst section 5 consider public awards and the choice of confidentiality. Section

\footnotetext{
${ }^{4}$ For a model of monitoring of decision makers through appeals see Iossa and Palumbo (2005).
} 
6 compares arbitration with litigation in court. Section 7 concludes.

\section{Related literature}

\section{On reputational concerns in decision making}

The paper is closely related to the recent literature on careerist decision makers, such as regulators, managers or experts who try to prove their ability to make the correct decision. In the context of dispute resolutions, Levy (2005) shows that careerist judges may contradict precedents too often in order to signal their ability, whilst in the context of policy making, Leaver (2004) argues that less able bureaucrats may use soft policies so as to keep interests groups quiet and mistakes out of public eyes. ${ }^{5}$ We contribute to this literature by showing how reputational concerns can lead to decision bias in arbitration. We also highlight the trade off between decision bias and information acquisition and how this trade-off is resolved depending on the degree of symmetry between the disputing parties. Finally, we explicitly analyze the role of confidentiality in reputational models of decision making, and thus the role of market monitoring.

\section{On revolving doors}

The paper is also related to the literature on revolving doors which analyzes how the possibility to obtain lucrative industry jobs affects the performance of regulators (or public officials in general). Post-regulatory employment may induce regulators to be lenient towards the industry if the industry rewards opportunistic behaviour with future employments. It may also result in regulators choosing overly-complex regulations so as to increase the value of their expertise for the industry (see Heyes, 2003). Revolving doors can however generate the positive effect of increasing incentives to invest in human capital that enhances qualifications for a postagency job at the firm (see Che, 1995; and Salant, 1995). In our paper, contrary to this literature, we focus on reputational issues and derive opportunistic behaviour from a desire to appear competent. As shown by Bloom and Cavanagh (1986), there is only weak evidence that parties reward "loyal" arbitrators by reappointing them. Evidence is much stronger that parties value experience in arbitrators and good qualifications.

\footnotetext{
${ }^{5}$ In these papers, as in ours, it is assumed that the quality of information is unverifiable ex post. The quality of information is instead generally assumed verifiable in the literature on experts' advice, where reputational concerns can generate biased advice (in the form of herding or anti-herding) as shown by Levy (2004), Bourjade and Jullien (2005) and Ottaviani and Soerensen (2006).
} 


\section{On arbitration}

Finally the paper is related to the literature on arbitration. Shavell (1995) discusses the choice between litigation and alternative-dispute resolutions (ADR), including arbitration, and suggests that ADR may be chosen because it may lower the cost of resolving disputes or engender superior incentives through greater accuracy of result. In most studies on arbitration, arbitrators are viewed as experts who are able to verify dimensions of commercial contracts better than court judges (see also example Dixit, 2003; and Chakravarty and Makris, 2005). These papers cannot explain why contractual parties often but not always choose to employ arbitration as a dispute-resolution process. They also do not discuss the role of confidentiality or of selection of arbitrators.

\section{The basic model}

The players and the general setting

There are two periods and four players, an arbitrator (he), two parties, $P_{1}$ (she) and $P_{2}$ (she), and a market player $M$ (it). At the beginning of period $1, P_{1}$ and $P_{2}$ enter a contractual relationship involving issues of technical complexity summarized by a parameter $\chi \in[0,1]$ and decide which dispute-resolution process to use in the event of a dispute. They have two options: arbitration and litigation in court. Under arbitration, the parties select the decision maker (i.e. the arbitrator) and choose whether to insert a confidentiality provision in the contract, appeals are not allowed. Under litigation in court, the decision-maker (i.e. the judge) is randomly selected, the dispute proceedings are public information and appeals are allowed. In this basic model we focus on the case of arbitration. We extend the basic model to study the case of litigation in court in Section 6.

During period 1 a dispute exogenously arises over the terms of an existing contract. There are two possible states, $\theta=1$ and $\theta=2$, and it is common knowledge that $\operatorname{Pr}(\theta=1)=1 / 2$. State $\theta=1$ corresponds to a situation where the interpretation of the contract is in the interest of $P_{1}$, whilst state $\theta=2$ corresponds to a situation where the interpretation of the contract is in the interest of $P_{2}$. There are only two possible decisions, $d \in\{1,2\}: P_{1}$ wins the dispute if $d=1$ whilst $P_{2}$ wins if $d=2$. The value of winning for a party is 1 . The 'correct' decision is $d=\theta$, which is interpreted as the decision that maximizes the joint surplus from the contractual relationship. 
Net of dispute-resolution costs, the parties' joint surplus from the contractual relationship is

$$
U=\operatorname{Pr}(d=\theta) u+\omega c
$$

where $u>0$ is the value of a correct decision; an incorrect decision is valued at zero. ${ }^{6} \omega$ is a dummy variable with $\omega=1$ in the presence of a confidentiality provision and $\omega=0$ otherwise. $c>0$ denotes the value of confidentiality for the parties stemming from the possibility that market knowledge of the dispute negatively impacts on the firm's reputation or share values.

The parties $P_{1}$ and $P_{2}$ can be either short-lived or long-lived. A long-lived party is more likely to need arbitral services again in the future than a short-lived party. Long-lived parties can be thought as corporations or parties with a large volume of affairs. Short-lived parties can be thought as individuals or small businesses. We assume that whether a party is longlived or short lived is common knowledge, and, unless stated otherwise, $P_{1}$ is long-lived whilst $P_{2}$ is short-lived. We capture the repeated interaction in a stylized way by assuming that in period 2, $P_{1}$ and $M$ need the service of an arbitrator with probability 1 , whilst $P_{2}$ does not need arbitral services.

\section{The information of the parties and of the market}

In modelling the information structure we wish to capture two central features of disputeresolution processes. First, the parties to a dispute are likely to have some information about the state of the world. Second, at the time the parties start the arbitral proceedings, they are likely to hold a positive view of their case, with $P_{i}$ assigning a higher probability to $\theta=i$ than $P_{j}$. Different priors is a standard assumption in the literature on dispute resolutions and it is one of the main explanations as to why parties litigate rather than settle. In the context of settlement bargaining with asymmetric information, it has been shown that settlement is more likely to fail when each party has a positive signal about the state of the world. Thus, when a case is not settled an inference can be drawn that each party is likely to have a positive view about the case (see for example Schweizer, 1989).

Formally, we assume that in each state $\theta, P_{i}$ observes a private and unverifiable signal $\sigma_{i} \in\{1,2\}$. The realization of the signal depends on the state $\theta$ and on the identity of the

\footnotetext{
${ }^{6}$ This formulation captures in a reduced form the idea that better enforcement of contractual terms helps to ensure better incentives for relationship-specific investments, which in turn increases the joint surplus from the contractual relationship. Whilst, this is true in general contexts, Anderlini, Felli and Postelwaite (2006) show that there can be instances where efficiency calls for the dipute-resolution process not to enforce the will of the parties expressed in the contract.
} 
party: $P_{i}$ is more likely to observe a positive signal $\sigma_{i}=i$ than a negative signal $\sigma_{i}=j ; P_{i}$ is also more likely to observe a positive signal in the state where the correct decision is the one in her interest (i.e. when $\theta=i$ ) than the one against (i.e. $\theta=j$ ). In particular, we assume that in state $i P_{i}$ always observes $\sigma_{i}=i$. In state $j$ she observes $\sigma_{i}=j$ with probability $\nu$ and $\sigma_{i}=i$ with probability $1-\nu$. That is: $\operatorname{Pr}\left(\sigma_{i}=i \mid \theta=i\right)=1, \operatorname{Pr}\left(\sigma_{i}=j \mid \theta=i\right)=0$, $\operatorname{Pr}\left(\sigma_{i}=j \mid \theta=j\right)=\nu$ and $\operatorname{Pr}\left(\sigma_{i}=i \mid \theta=j\right)=1-\nu$.

Accordingly, when $P_{i}$ observes a positive signal, her posterior belief that $\theta=i$ is $\operatorname{Pr}(\theta=$ $\left.i \mid \sigma_{i}=i\right)=\frac{1}{2-\nu}$, whilst if $P_{i}$ observes a negative signal, she is certain that the state is $j$ : $\operatorname{Pr}\left(\theta=i \mid \sigma_{i}=j\right)=0$. Thus, $\nu$ captures the precision of the information in the hands of the parties. When $\nu=0$, parties are uninformed: each party observes $\sigma_{i}=i$ regardless of the state of the world. When $\nu=1$, parties are perfectly informed; observing $\sigma_{i}=i$ reveals to $P_{i}$ that the state is $\theta=i$, whilst observing $\sigma_{i}=j$ reveals to $P_{i}$ that the state is $\theta=j$.

$M$ has no private information. It observes $d$ if the award is public; if the award is confidential it observes nothing.

The competency and the information of the arbitrator

At the outset the arbitrator has no information. However, before taking a decision he can choose to exert costly and unobservable effort into acquiring information about the state of the world $\theta$. The precision of the information acquired depends on the ability of the arbitrator. For simplicity, we assume that there are only two types $t$ of arbitrators: the competent $(t=C)$ and the incompetent $(t=I)$. If type $C$ exerts effort, he observes $\theta$ with probability 1 , otherwise he observes nothing. Instead, type $I$ observes nothing no matter how hard he tries. The model can be generalized to continuous types (as in Levy 2005). The cost of acquiring information in terms of disutility of effort is $\Psi \in[0,1]$. $\Psi$ is distributed according to a cumulative distribution function $F(\Psi, \chi)$, with $F_{\chi}(\Psi, \chi)<0$ : the more technical the subject matter the higher the expected disutility of effort; we also assume that $F_{\chi \Psi} \geq 0$, which implies that greater technical complexity raises the expected disutility of effort at a decreasing rate.

We denote by $e^{t}$ the effort exerted by the arbitrator of type $t$, with $e^{t}=1$ if the arbitrator exerts effort and $e^{t}=0$ otherwise. Since it is immediate that $e^{I}=0$, we shall focus on effort of type $C$. The arbitrator knows his type, whilst $P_{1}$ and $P_{2}$, and $M$ only know that the fraction of type $C$ is given by $\gamma \in(0,1)$. For simplicity and without loss of generality 
we assume that $\Psi$ is realized after a dispute has arisen and that the realized value of $\Psi$ is observable. Relaxing this assumption would just add an additional parameter.

\section{The objectives of the arbitrator and selection of arbitrators}

In expression (1), we have seen that parties value dispute-resolution processes where correct decisions are reached more often. We will show later in the paper that more competent arbitrators are more likely to make correct decisions. This implicitly rationalizes a relationship between the earning prospect of an arbitrator and his reputation for competency. ${ }^{7}$

To capture the reputational concerns of the arbitrator in a simple way, we assume that the arbitrator's income is equal to the belief held by the arbitrator's 'employer' that the arbitrator is competent. Thus, the arbitrator's income in period 1 is equal to $\gamma$, and since period 1-income plays no role, it will be disregarded.

In period 2 , the arbitrator can be hired either by $M$ or by the long-lived party $P_{1}$. $P_{1}$ and $M$ update their beliefs rationally (being short-lived, the belief of $P_{2}$ plays no role). We denote by $\alpha_{\sigma_{1}}^{d} P_{1}^{\prime} s$ posterior belief that the arbitrator is competent upon observing signal $\sigma_{1}$, decision $d$, and given $P_{1}^{\prime} s$ conjecture about the strategy of $C$ and $I$. The posterior belief of $M$ depends on whether there is confidentiality. Under confidentiality, $M$ does not observe the decision and therefore its posterior belief is equal to the prior $\gamma$. When instead the decision is public information the posterior belief of $M$, given a decision $d$ and $M^{\prime} s$ conjecture about the strategy of $C$ and $I$, is denoted by $\gamma^{d}$. We then have that income in period 2, denoted by $y$, is equal to the highest posterior belief of $P_{1}$ and $M$. This reduced form captures the idea that the belief of $M$ affects the reservation income of an arbitrator, whilst the belief of $P_{1}$ affects the fee $P_{1}$ is willing to pay. ${ }^{8}$

Assuming no discounting, the payoff of the arbitrator when he is type $t$ is then given by

$$
\Pi=y-e^{t} \Psi^{t}
$$

where

$$
y= \begin{cases}\max \left\{\alpha_{\sigma_{1}}^{d}, \gamma\right\} & \text { if there is confidentiality } \\ \max \left\{\alpha_{\sigma_{1}}^{d}, \gamma^{d}\right\} & \text { if the award is public information }\end{cases}
$$

\footnotetext{
${ }^{7}$ We disregard the possibility that the arbitrator has outcome concerns since outcome concerns play a limited role in what follows. In particular, they only serve to ensure that no pooling equilibrium exists.

${ }^{8}$ Any bargaining process where $P_{1}$ does not have full bargaining power would lead to an equilibrium fee higher than the reservation fee.
} 


\section{Timing}

Period 1. At the beginning of period $1, P_{1}$ and $P_{2}$ enter a contractual relationship involving technical issues of a dimension summarized by $\chi$, and agree on whether to employ arbitration or litigation in court. If they agree on arbitration they choose whether to opt for confidentiality.

A dispute then arises with unobservable state of the world $\theta$, and $\Psi$ is realized. $P_{1}$ and $P_{2}$ observe private signals $\sigma_{1}$ and $\sigma_{2}$ and the competent arbitrator observes $\theta$ if he chooses $e^{C}=1$, and nothing otherwise. The incompetent arbitrator observes nothing.

The arbitrator chooses $d$, observed by $P_{1}$ and $P_{2}$, and by $M$ if the award is public. $P_{1}$, $P_{2}$ and $M$ update their beliefs.

Period 2. The arbitrator receives $\Pi$, as defined in (2) and the disputing parties receive their payoffs.

For simplicity there is no discounting.

\section{Confidential award}

\subsection{The payoffs}

We start by considering the case of confidentiality. We proceed as follows. First we study the behaviour of $C$ and of $I$, assuming that $C$ has acquired information on $\theta$. Then, in Section 4.3 , we analyze the incentives for $C$ to acquire information.

When the award is confidential, $M^{\prime} s$ posterior belief that the arbitrator is competent is equal to the prior $\gamma$. Thus the arbitrator can always obtain an income of at least $\gamma$ in period 2 . The arbitrator will obtain an income higher than $\gamma$ only if $P_{1}^{\prime} s$ posterior belief $\alpha_{\sigma_{1}}^{d}$ is greater than $\gamma$. In particular, if the arbitrator observes $\theta=i$ and makes a decision $d$, his expected income is given by

$$
y=\operatorname{Pr}\left(\sigma_{1}=1 \mid \theta=i\right) \max \left\{\gamma, \alpha_{1}^{d}\right\}+\operatorname{Pr}\left(\sigma_{1}=2 \mid \theta=i\right) \max \left\{\gamma, \alpha_{2}^{d}\right\}
$$

Instead, if the arbitrator has no information on the realization of $\theta$, his expected income is

$$
y=\operatorname{Pr}\left(\sigma_{1}=1\right) \max \left\{\gamma, \alpha_{1}^{d}\right\}+\operatorname{Pr}\left(\sigma_{1}=2\right) \max \left\{\gamma, \alpha_{2}^{d}\right\}
$$

Let us now analyze the behavior of $C$. When $C$ observes $\theta=1$, he knows that $P_{1}$ can only have observed $\sigma_{1}=1$, since $\operatorname{Pr}\left(\sigma_{1}=1 \mid \theta=1\right)=1$. His expected income in period 2 is 
therefore $y=\max \left\{\gamma, \alpha_{1}^{d}\right\}$ and if $C$ chooses $d=i$ his expected payoff is

$$
V(d=i \mid \theta=1)=\max \left\{\gamma, \alpha_{1}^{i}\right\}
$$

Now consider the case where $C$ observes $\theta=2$. Here $C$ knows that $P_{1}$ has observed $\sigma_{1}=1$ with probability $(1-\nu)$ and $\sigma_{1}=2$ with probability $\nu$ since $\operatorname{Pr}\left(\sigma_{1}=1 \mid \theta=2\right)=1-\nu$, and $\operatorname{Pr}\left(\sigma_{1}=1 \mid \theta=2\right)=\nu$. Thus, the expected payoff of $C$ if he chooses $d=i$ is

$$
V(d=i \mid \theta=2)=(1-\nu) \max \left\{\gamma, \alpha_{1}^{i}\right\}+\nu \max \left\{\gamma, \alpha_{2}^{i}\right\}
$$

Finally, consider the behavior of $I$. Since $I$ has no information on the state of the world, his expected income is given by (4), for any given decision $d$. Using $\operatorname{Pr}\left(\sigma_{1}=1\right)=\frac{1}{2}(2-\nu)$ and $\operatorname{Pr}\left(\sigma_{1}=2\right)=\frac{1}{2} \nu$, it follows that if $I$ chooses $d=i$ his expected payoff is

$$
V(d=i)=\frac{1}{2}\left[(2-\nu) \max \left\{\gamma, \alpha_{1}^{i}\right\}+\nu \max \left\{\gamma, \alpha_{2}^{i}\right\}\right]
$$

\subsection{Equilibrium}

In this section we analyze the decision-making behaviour of arbitrators when the award is confidential. Pooling equilibria are of limited interest and will be disregarded. ${ }^{9}$ In what follows we let superscripts $l l, l s, s s$ denote respectively the cases with two long-lived parties, only one, or none.

Proposition 1 Let $e^{C}=1$. Under arbitration with a confidentiality provision, when one party to the dispute is long-lived whilst the other party is short-lived, at the equilibrium a decision bias in favour of the long-lived party arises. In particular, the competent arbitrator chooses $d=\theta$ whilst the incompetent arbitrator chooses $d=1$ with probability $\widehat{z}^{l s}$, where $\widehat{z}^{l s}=\frac{1}{2}$ for $\nu=0,1$ and $\widehat{z}^{l s} \in\left(\frac{1}{2}, 1\right)$ for $\nu \neq 0,1$.

Proof: see the Appendix.

\footnotetext{
${ }^{9}$ It is sufficient for the arbitrator to have minimal outcome concerns that pooling equilibria do not exist. To see this, let $\mu$ denote the benefit for the arbitrator from taking the correct decision and consider the case where both $C$ and $I$ choose $d=i$. Then using Bayes rule we have: $\alpha_{1}^{i}=\alpha_{2}^{i}=\gamma$. In this case when $C$ observes $\theta=j$, if he chooses $d=i$ he obtains a payoff of $\gamma$, whilst if he chooses $d=j$, he obtains

$$
V(d=j \mid \theta=j)=\mu+\left[\operatorname{Pr}\left(\sigma_{1}=1 \mid \theta=j\right) \max \left\{\gamma, \alpha_{1}^{j}\right\}+\operatorname{Pr}\left(\sigma_{1}=2 \mid \theta=j\right) \max \left\{\gamma, \alpha_{2}^{j}\right\}\right]
$$

which is greater than $\gamma$.
} 
The equilibrium involves partial separation of types. The way for $C$ to transmit information about his type in the clearest possible way is to select a different decision for any different $\theta$, as $P_{1}$ has some information on $\theta$. Ruling out mirror equilibria, this leads $C$ to choose $d=\theta$. $I$, being uninformed, cannot do the same. He then biases his decision in favour of $P_{1}$ with the aim to appear competent to her and enhance his earning prospects. $I$ anticipates that since $P_{1}$ has a biased prior (namely she is more likely to observe $\sigma_{1}=1$ than $\sigma_{1}=2$ ), she is more likely to (mistakenly) take a decision $d=1$ as correct. At the equilibrium $I$ then mixes between the two decisions, choosing $d=1$ with probability greater than $1 / 2$. An equilibrium where $I$ chooses $d=1$ with probability 1 cannot arise since a decision $d=2$ would then convey competence and give incentives for $I$ to deviate.

There are only two cases where the decision bias does not arise: when the parties are uninformed, $\nu=0$, and when the parties are perfectly informed, $\nu=1$. In both cases the belief of $P_{1}$ following a decision $d$ are symmetrical and the only equilibrium has $\widehat{z}^{l s}=1 / 2$.

Proposition 1 has shown that reputational concerns create a decision bias in favour of $P_{1}$; the corollary below highlights how this bias is ultimately due to the parties being asymmetric.

Corollary 1 With symmetric parties, i.e. when parties are either both long-lived or both short lived, there is no decision bias under arbitration:

$$
\widehat{z}^{l l}=\widehat{z}^{s s}=\frac{1}{2} .
$$

Proof: see the Appendix.

\subsection{Information acquisition}

Suppose now that $e^{C}=0$. In this case $C$ has no information about the state of the world and his behavior is the same as the behaviour of $I$. We can therefore calculate the value of information for $C$ by comparing the expected payoff of $C$ when $e^{C}=1$ with the expected payoff of $I$.

Proposition 2 Under arbitration with a confidentiality provision, when one party to the dispute is long-lived whilst the other party is short-lived, the value of information for the competent arbitrator is given by

$$
\widehat{\Psi}^{l s}=\frac{1}{2}\left(\alpha_{1}^{1}(.)-\gamma\right)
$$


where $\alpha_{1}^{1}()=.\frac{\gamma}{\gamma+(2-\nu)(1-\gamma) \bar{z}^{l s}}$ and where $\widehat{\Psi}^{l s}($.$) is increasing in the precision of information$ of the parties, $\nu$, and decreasing in the decision bias $\left(\widehat{z}^{l s}-\frac{1}{2}\right)$. The competent arbitrator exerts effort into information acquisition for all $\Psi \leq \widehat{\Psi}^{l s}$.

Proof: see the Appendix.

In principle, arbitration rules could be designed in such a way as to take away the control by the parties over the selection of the arbitrator so as to eliminate the decision bias. ${ }^{10}$ However, this effect comes at a cost since reputational concerns create the incentives of the arbitrator to exert effort in information acquisition. Expression (8) in fact shows that information is valuable to $C$ as it helps to increase his future earning prospect above market rate $\gamma$ by an amount proportional to the posterior belief of $P_{1}, \alpha_{1}^{1}$.

Intuitively, $C$ has incentives to appear competent to $P_{1}$. This is easier if $C$ has information about $\theta$ because the greater ability to acquire information is the advantage that $C$ has over $I$. By observing $\theta, C$ can predict better than $I$ the realization of the signal $\sigma_{1}$ and therefore the information that $P_{1}$ holds about the state of the world. This helps $C$ taking the (correct) decision that signals his competency to $P_{1}$. Reputational concerns with $M$ have instead no impact on the effort of the arbitrator since $M$ is uninformed about $\theta$ and cannot observe the decision.

Proposition 2 also shows that $C^{\prime} s$ effort increases with the precision of the information held by $P_{1}$ (as captured by $\nu$ ). Intuitively, as $\nu$ increases, the likelihood that $P_{1}$ will indeed perceive as correct the decision $d=\theta$ made by $C$ also increases. A greater decision bias (higher $\widehat{z}^{l s}$ ) instead reduces the effort in information acquisition, by lowering the posterior belief of $P_{1}$ when $d=1$ and $\sigma_{1}=1$.

Consider now how effort in information acquisition changes with the identity of the parties in the dispute. The proposition below suggests that not only that reputational concerns with the parties create incentives for the arbitrator to acquire information, but also that the relationship is monotone.

Proposition 3 The incentives for the arbitrator to acquire information are greatest when both parties in the dispute are long-lived and lowest when both parties in the dispute are

\footnotetext{
${ }^{10}$ This is for example what the LCIA has done.
} 
short-lived:

$$
\begin{gathered}
\widehat{\Psi}^{l l}>\widehat{\Psi}^{l s}>\widehat{\Psi}^{s s} \\
\text { with } \\
\widehat{\Psi}^{l l}=\alpha_{1}^{1}\left(\widehat{z}^{l s}\right)-\gamma \\
\widehat{\Psi}^{s s}=0
\end{gathered}
$$

where $\alpha_{1}^{1}\left(\widehat{z}^{l s}\right)=\frac{\gamma}{\gamma+(2-\nu)(1-\gamma) \widehat{\bar{z}}^{l s}}$.

Proof: See the Appendix.

Note that when the realized level of $\Psi$ is greater than $\widehat{\Psi}$, it is public information that the arbitrator will not have sufficient incentives to acquire information and arbitration performs very poorly. What exactly happens in this case is irrelevant to our analysis; it could be that the parties renegotiate and decide to go to court, or that they throw a coin. For simplicity we assume that when $\Psi>\widehat{\Psi}$ each party wins with probability $\frac{1}{2}$. It follows that the ex-ante payoff of the parties under arbitration with a confidentiality provision is given by

$$
\widehat{U}^{k}=\frac{1}{2}\left(1+F\left(\widehat{\Psi}^{k}, \chi\right) \gamma\right) u+c ; \quad k=l l, l s, s s
$$

which in light of Proposition 3 yields the following corollary.

Corollary 2 The surplus of the parties from employing arbitration as dispute-resolution mechanism is greatest when both parties in the dispute are long-lived and lowest when both parties in the dispute are short-lived:

$$
\widehat{U}^{l l}(\gamma, \nu)>\widehat{U}^{l s}(\gamma, \nu)>\widehat{U}^{s s}(\gamma, \nu)
$$

Remark 1 We have assumed that the parties suffer a loss from an incorrect decision but we have ruled out the possibility that a decision bias is harmful to the parties per se. However, in practice the decision bias can indeed reduce the joint surplus from the contractual relationship, when for example it is costly to raise funds to compensate the short-lived party for the future unfair division of the surplus that the decision bias will generate. If we introduced an explicit cost of decision bias, the case for arbitration with asymmetric parties would be further reduced. Our qualitative results would however remain unchanged. 


\section{Choosing confidentiality}

Now suppose that the parties choose to opt for a public award. The results are qualitatively similar to the ones under confidentiality provision, the only difference stemming from $M$ now being able to observe the decision made by the arbitrator and update its belief accordingly, so that $y=\max \left\{\alpha_{\sigma_{1}}^{d}, \gamma^{d}\right\}$.

Proposition 4 Let $e^{C}=1$. Under arbitration, with public proceedings, when one party to the dispute is long-lived whilst the other party is short-lived, at the equilibrium a decision bias in favour of the long-lived party arises. The competent arbitrator always chooses $d=\theta$ whilst the incompetent arbitrator chooses $d=1$ with probability $\widetilde{z}^{l s}$, where $\widetilde{z}^{l s}=\frac{1}{2}$ for $\nu=0,1$ and $\widetilde{z}^{l s} \in\left(\frac{1}{2}, 1\right)$ for $\nu \neq 0,1$. Compared to the case of confidentiality, the decision bias is reduced: $\widetilde{z}^{l s} \leq \widehat{z}^{l s}$.

Proof: see the Appendix.

The decision bias in favour of the long-lived party is greater when the award is confidential than when it is public. Since in equilibrium it is type $I$ who biases his decision in favour of $P_{1}, M^{\prime} s$ posterior belief that the arbitrator is competent is higher when $M$ observes a decision $d=2$ than when he observes $d=1$ (i.e. $\gamma^{1}>\gamma^{2}$ when $z>\frac{1}{2}$ ). Other things equal, this decreases the expected gain of $I$ from taking a decision $d=1$ and thus his incentives to bias his decision in favour of the long-lived party.

We can now calculate how the incentives of $C$ to acquire information change with a public award.

Lemma 1 Under arbitration, in the absence of a confidentiality provision, the value of information for the arbitrator is given by

$$
\begin{aligned}
\widetilde{\Psi}^{l l}(\gamma, \nu) & =\widehat{\Psi}^{l l} \\
\widetilde{\Psi}^{l s}(\gamma, \nu) & =\frac{1}{2}\left(\alpha_{1}^{1}\left(\widetilde{z}^{l s}\right)-\gamma^{2}\left(\widetilde{z}^{l s}\right)\right) \\
\widetilde{\Psi}^{l s}(\gamma, \nu) & =\widehat{\Psi}^{s s}
\end{aligned}
$$

where $\alpha_{1}^{1}\left(\widetilde{z}^{l s}\right)=\frac{\gamma}{\gamma+(2-\nu)(1-\gamma) \widetilde{z}^{l s}}$ and $\gamma^{2}\left(\widetilde{z}^{l s}\right)=\frac{\frac{1}{2} \gamma}{\frac{1}{2} \gamma+(1-\gamma)\left(1-\widetilde{z}^{l s}\right)}$. Compared to the case of confidentiality, incentives to acquire information are stronger: $\widetilde{\Psi}^{k}(.) \geq \widehat{\Psi}^{k}($.$) .$ 
Proof: see the Appendix.

As under confidentiality, with public proceedings the incentives for $C$ to acquire information stem from the reputational concerns of the arbitrator. However, the absence of decision bias increases the incentives for $C$ to acquire information. Given that in case $l s$ the bias is smaller when the award is public than when it is confidential, incentives to acquire information are greater when the award is public. In the remaining cases, $l l$ and $s s$, confidentiality has no impact on the effort of the arbitrator.

In light of Proposition 4 and Lemma 1, the ex ante payoff of the parties under public proceedings is

$$
\widetilde{U}^{k}=\frac{1}{2}\left(1+F\left(\widetilde{\Psi}^{k}, \chi\right) \gamma\right) u ; k=l l, l s, s s
$$

and, conditional on the parties employing arbitration in the event of a dispute, the parties will choose to insert a confidentiality provision in the contract if

$$
\widehat{U}^{k} \geq \widetilde{U}^{k}
$$

where $\widehat{U}^{k}$ and $\widetilde{U}^{k}$ are given by (9) and (10), respectively. From Lemma 1 and from differentiating the difference between $\widehat{U}$ and $\widetilde{U}$ with respect to $\chi$, we obtain the following proposition.

Proposition 5 (i) Conditional on the parties using arbitration, the incentives of the parties to choose confidentiality are greater for symmetric parties, than when the parties are asymmetric. (ii) Lower technical complexity decreases incentives for confidentiality.

Proof: see the Appendix.

We have seen in Lemma 1 that confidentiality reduces the effort of the arbitrator when parties are asymmetric. When the subject matter is complex, providing incentives to $C$ to acquire information is critical. This explains point (i). To increase incentives for information acquisition, asymmetric parties may then choose to give up confidentiality. Instead, when parties are both long-lived or are both short-lived there is no decision bias and the effort of the arbitrator is unaffected by the presence of a confidentiality provision. Confidentiality will then optimally be chosen whenever arbitration is chosen, which explains point (ii). 


\section{Arbitration versus Court}

\subsection{Selection of arbitrators by the parties}

In this section we compare arbitration with litigation in court, where litigation in court is identified as a dispute-resolution process in which the decision-maker (i.e., the judge) is randomly selected, the dispute proceedings are public information and appeals are allowed. Since the parties in the dispute play no role in the selection of the judge, there is no role for reputational concerns with the parties under litigation in court. ${ }^{11}$

However, the judge cares about appearing competent to $M$. We capture the judge's reputational concerns by assuming that the judge's income is equal to the posterior belief of $M$, following a decision $d$ and any information that comes through the appeals.

To study the role of appeals, we modify the basic model as follows. We assume that there is an appeals court that is able to find verifiable evidence of the correct decision with probability $r$, whilst with probability $(1-r)$ it finds nothing and confirms the initial decision. The parameter $r$ captures in a simple way the efficacy of the appeal system. Whether the appeals court finds evidence is observable. Thus there is no agency problem with the appeals court, which allows us to focus on the incentives of the lower-court judge.

Recourse to appeal is costly for the party seeking an appeal; the cost is a random variable $H$ distributed uniformly over the interval $[0,1]$. We assume that $H$ is realized only after an initial decision is made, which implies that the judge views appeals as uncertain. Relaxing this assumption has no qualitative impact on our results; it only adds an additional parameter. The following proposition then summarizes the characteristics of the equilibrium under litigation in court.

Proposition 6 Let $e^{C}=1$. Under litigation in court, at the equilibrium $C$ chooses $d=\theta$, whilst type I randomizes by choosing $d=1$ with probability $\bar{z}$ and $d=2$ with probability $1-\bar{z}$. There is no decision bias: $\bar{z}=\frac{1}{2}$ regardless of whether the parties in the dispute are short lived or long lived.

Take the behaviour of the losing party. Upon observing a decision $d=i, P_{j}$ never goes to trial if she has observed a negative signal $\sigma_{j}=i$, since she knows that $\operatorname{Pr}\left(\theta=j \mid \sigma_{j}=i\right)=0$.

\footnotetext{
${ }^{11}$ We are implicitly ruling out the possibility that the judge in period 1 become an arbitrator in period 2. Relaxing this assumption would have no qualitative impact on our results concerning the choice between arbitration and litigation in court, although it could introduce a decision bias in favour of the long-lived party also under litigation in court.
} 
Instead, $P_{j}$ may gain from appealing if she has observed a positive signal $\sigma_{j}=j$. In particular, $P_{j}$ will choose to appeal if she has sufficiently high expectations to reverse the initial decision, that is if

$$
r \operatorname{Pr}\left(\theta=j \mid \sigma_{j}=j, d=i\right)-H \geq 0
$$

The incentive of the losing party to appeal increases in $r$ and decreases in the cost of an appeal, $H$. For all $H \leq \bar{H}^{i}$, where, using Bayes rule and players' equilibrium strategies,

$$
\bar{H}^{i} \equiv r \operatorname{Pr}\left(\theta=j \mid \sigma_{j}=j, d=i\right)=\left\{\begin{array}{cl}
\frac{r(1-\gamma) \bar{z}}{\gamma(1-\nu)+(2-\nu)(1-\gamma) z} & \text { if } d=1 \\
\frac{r(1-\gamma)(1-z)}{\gamma(1-\nu)+(2-\nu)(1-\gamma)(1-z)} & \text { if } d=2
\end{array}\right.
$$

the losing party will appeal if and only if she has observed a positive signal. For all $H>\bar{H}^{i}$, the losing party never appeals.

Now consider the payoff of type $C$ and the role that the information conveyed through the appeals process plays in shaping the reputation of the judge. Note that frequency of reversal on appeals plays indeed a significant part in judicial promotion in almost all countries (see e.g. Miceli and Cosgel, 1994).

Let $C$ choose $d=\theta$. With probability $\bar{H}^{i}, H \leq \bar{H}^{i}$ is realized and the losing party appeals if and only if she has observed a positive signal. Since $d=\theta$, in appeal, $C^{\prime} s$ decision is proven correct with probability $r$, and, through Bayesian updating $M^{\prime} s$ posterior belief is given by

$$
\bar{\gamma}^{i}=\frac{\gamma}{\gamma+(1-\gamma) z}
$$

With probability $1-r$, the appeals court does not observe any verifiable information and the initial decision is automatically confirmed. In this case, however, the signal of the losing party is inferred by $M$ through her decision to appeal. Thus $M^{\prime} s$ posterior beliefs, following a decision $d=i$ is

$$
\overline{\bar{\gamma}}^{i}=\frac{\gamma(1-\nu)}{\gamma(1-\nu)+(2-\nu)(1-\gamma) z}
$$

Finally, when $H>\bar{H}^{i}$ is realized, which occurs with probability $\left(1-\bar{H}^{i}\right)$, appeals costs are so high that under no circumstances will the losing party consider to appeal. Thus no information about the correctness of $C^{\prime} s$ decision is revealed.

In light of the above, the payoff of $C$ from choosing $d=\theta$ is

$$
V^{C}(d=i \mid \theta=i)=\bar{H}^{i}\left(r \bar{\gamma}^{i}+(1-r) \overline{\bar{\gamma}}^{i}\right)+\left(1-\bar{H}^{i}\right) \gamma^{i}
$$


with $\gamma^{i}$ given by (15).

Consider now the payoff of $I$ from randomizing between decisions. With probability $\frac{1}{2}, I$ takes the correct decision and obtains the same payoff as $C$. With probability $\frac{1}{2}, I^{\prime} s$ decision is incorrect. In this case, with probability $r \bar{H}^{i}$, the decision is reversed in appeal and $I^{\prime} s$ type is fully revealed. In the remaining case, $M^{\prime} s$ posterior beliefs is the same as in the discussion above for type $C$ and thus is given by (11). It follows that the expected payoff of $I$ when he chooses $d=i$ is

$$
V^{I}(d=i)=\frac{1}{2} V^{C}(d=i \mid \theta=i)+\bar{H}^{i}(1-r) \overline{\bar{\gamma}}^{i}+\frac{1}{2}\left(1-\bar{H}^{i}\right) \gamma^{i}
$$

By equating $V^{I}(d=1)$ to $V^{I}(d=2)$, we obtain $\bar{z}=\frac{1}{2}$. The difference between $E V^{C}$ and $E V^{I} \equiv V^{I}(d=1)$ gives the value of information under litigation in court

$$
\bar{\Psi}=\frac{1}{2} r \bar{H}^{1} \bar{\gamma}^{1}
$$

In light of this, the ex ante payoff of the parties under litigation in court in case $k$ is

$$
\bar{U}(r)=\left[\frac{1}{2}(1+F(\bar{\Psi}, \chi) \gamma)+\frac{1}{2}(1-\gamma) r \bar{H}^{1}\left(r-\bar{H}^{1}\right)\right] u
$$

and it is immediate that the utility of the parties is independent of whether they are long-lived or short lived, since period 2 plays no role. The following proposition is then obtained.

Proposition 7 (i) There exists a cutoff value of $r$, denoted by $r^{k}(c)$ such that for $r \leq r^{k}(c)$ the parties prefer arbitration to litigation in court, whilst for $r>r^{k}(c)$, the opposite holds. (ii) $r^{k}(c)$ is non-decreasing in $c$, that is parties are more likely to prefer arbitration to litigation in court when the value of confidentiality is high; (iii) $r^{l l}(c)<r^{l s}(c)<r^{s s}(c)$, the parties are most likely to choose arbitration when they are both long-lived whilst they are least likely to choose arbitration when they are both short lived.

Proof: see the Appendix.

To highlight the role of selection of arbitrators by the parties as opposed to random selection of judges under litigation in court, consider the case where there are no appeals under litigation in court. This occurs when $r=0$. In this case, the incentives to acquire information are weaker under litigation in court than under arbitration because of the incentives that reputational concerns with the parties generate under arbitration. For this reason the parties prefer arbitration to litigation in court when $r=0$. 
Now consider the opposite case, where appeals take place and are perfectly informative, as is the case when $r=1$. Appeals improve the quality of the decision under litigation in court for two reasons. First, if there is an appeal, the correct decision is found with probability $r=1$. Second, if there is no appeal but the cost of an appeal is low, it is inferred that the losing party had a pessimistic view as to the probability of winning, which perfectly reveals her signal. Because of these two reasons, the decision maker is subject to a better assessment of the quality of his decision than in a system such as arbitration where there are no appeals. Bing the assessment symmetric, there is also no decision bias. Finally, under litigation in court the incompetent decision maker faces the threat of reversal in appeal where reversal perfectly revels his incompetency, whereas under arbitration, the arbitrator's incompetency is never perfectly revealed to the market. For this reason incentives to acquire information are higher under litigation in court than under arbitration, and parties prefer litigation in court to arbitration.

Cases where $r \in(0,1)$ fall between these two extremes. Then we obtain two results. First, arbitration is more likely to be chosen when the value of confidentiality is high (point (ii) in Proposition 7). This is because the parties can always choose confidentiality under arbitration, whilst they cannot choose it under litigation in court. Second, arbitration is most likely to be chosen when parties are both long-lived parties (point (iii) in Proposition 7). This follows from the identity of the parties (short-lived vs. long-lived) playing no role under litigation in court, whilst it does play a role under arbitration (Corollary 1).

\section{Conclusion}

In this paper we have argued that the three main features of arbitration, namely, selection of arbitrators by the parties, finality of the decision and confidentiality, may result in biased and uninformed decision making when the parties to the dispute are asymmetric. In this respect the paper predicts that asymmetric parties will be, ceteris paribus, less inclined to choose arbitration as their dispute-resolution mechanism than symmetric and long-lived parties. However, selection of arbitrators by the parties has beneficial effects since it compensates for the lack of an appeals mechanism as monitoring device for arbitrators. Its effects are the highest when both parties to the disputes are long lived.

Our results help to explain the role for arbitration institutions. Arbitration institutions 
have created a market for their services by developing mechanisms for selecting and monitoring their recommended arbitrators so as to reduce problems of bias (for an extensive discussion, see Drahozal, 2001). Some institutions have even chosen explicitly to be in charge of appointing arbitrators (for example the LCIA). In any case, the list of arbitrators held by the arbitration institution is normally consulted by the parties, and the institutions update and change their list of arbitrators on a regular basis. Arbitration institutions also require their arbitrators to disclose any information that might be relevant to the standards of neutrality, including service as a neutral in any past or pending case involving any of the parties (see e.g. ICC Rules of Arbitration or AAA's National Rules for the Resolution of Employment Disputes). Failure to abide by these rules can expose the award to challenge, and jeopardize the reputation of the arbitrator. Also according to the AAA Code of Ethics for Arbitrators (2004), for example, for a reasonable period of time after the decision of a case, arbitrators should avoid entering into any business, professional, or personal relationship with any of the parties to the disputes. Failure to comply is likely to lead to the arbitrator being struck off the institutional list of recommended arbitrators. ${ }^{12}$ An explicitly modelling of the role of arbitral institutions could constitute an interesting scope for future research.

Our results also highlight the importance of leaving the parties free to choose the disputeresolution process. With voluntary arbitration the nonrepeated players (the short-lived party) has the option to insist on an unbiased dispute-resolution process, whilst with mandatory arbitration he does not. The risk of bias embedded in the use of mandatory arbitration has long been recognized by legal scholars, see for example Fitz (1999).

A possibility not analyzed in this paper is the use of tripartite arbitral panels. Practitioners tend to recommends the use of tripartite panels for disputes with a great financial stake, and the use of a sole arbitrator otherwise. This suggests that incentives to make the correct decision may be stronger when the number of arbitrators increases. However, the incentives to acquire information of an individual arbitrator may be weaker, because of free riding. A full analysis of decision making by tripartite arbitral panels could constitute an interesting scope for future research.

\footnotetext{
${ }^{12}$ Legal scholars have also suggested (see Bingham 1998) to increase the pool from which arbitrators may be selected so as to render their economic interest less direct, or to adopt random assignment of arbitrators to lists so as to reduce the likelihood of repeat appearance on each list of any single arbitrator. In Thomas v. Workmen's Compensation App. Board, 680 A.2d 24 (Pa. Comm. 1996), the court used the fact that an arbitator was randomly assigned to hear the case as tending to show there was no bias.
} 


\section{Appendix}

Proof of Proposition 1. The proof uses a series of Lemmas.

Lemma 2 Let $e^{C}=1$. Under arbitration with a confidentiality provision, at the equilibrium (i) type I uses mixed strategies, implying

$$
\nu\left[\max \left\{\gamma, \alpha_{2}^{2}\right\}-\max \left\{\gamma, \alpha_{2}^{1}\right\}\right]=(2-\nu)\left[\max \left\{\gamma, \alpha_{1}^{1}\right\}-\max \left\{\gamma, \alpha_{1}^{2}\right\}\right]
$$

and (ii) type $C$ always chooses $d=\theta$.

Proof of Lemma 2. (i) Suppose by contradiction that $I$ chooses $d=i$ with probability 1. Using Bayes rule this implies $\alpha_{1}^{i}, \alpha_{2}^{i}<1$ and $\alpha_{1}^{j}=\alpha_{2}^{j}=1$. Therefore $I$ obtains

$$
\begin{aligned}
{\left[\operatorname{Pr}\left(\sigma_{1}=1\right) \max \left\{\gamma, \alpha_{1}^{i}\right\}+\operatorname{Pr}\left(\sigma_{1}=2\right)\left\{\gamma, \alpha_{2}^{i}\right\}\right] \text { if } d } & =i \\
1 \text { if } d & =j
\end{aligned}
$$

and $I$ has incentives to deviate and choose $d=j$. It follows that in equilibrium $I$ must strictly randomize between $d=1$ and $d=2$. Setting $V(d=1)=V(d=2)$, using (7) yields expression (??).

(ii) By rearranging terms we can rewrite (??) as

$$
\left[(1-\nu) \max \left\{\gamma, \alpha_{1}^{2}\right\}+\nu \max \left\{\gamma, \alpha_{2}^{2}\right\}\right]-\left[(1-\nu) \max \left\{\gamma, \alpha_{1}^{1}\right\}+\nu \max \left\{\gamma, \alpha_{2}^{1}\right\}\right]=\left[\max \left\{\gamma, \alpha_{1}^{1}\right\}-\max \left\{\gamma, \alpha_{1}^{2}\right\}\right]
$$

which in light of (5) and (6) suggests that if $L H S=R H S>0$, then $C$ always strictly prefers $d=\theta$ to $d \neq \theta$. If $L H S=R H S<0$, then $C$ prefers to choose $d=\theta$ for $d \neq \theta$. The case of $d \neq \theta$ is a mirror equilibrium which we disregard.

From Lemma 2, we can calculate the posterior belief of $P_{1}$. Using Bayes rule and the arbitrator's equilibrium strategies, we have

$$
\begin{gathered}
\alpha_{1}^{1}=\frac{\gamma}{\gamma+(2-\nu)(1-\gamma) z} ; \alpha_{2}^{1}=0 \\
\alpha_{1}^{2}=\frac{\gamma(1-\nu)}{\gamma(1-\nu)+(2-\nu)(1-\gamma)(1-z)} ; \alpha_{2}^{2}=\frac{\gamma}{\gamma+(1-\gamma)(1-z)}
\end{gathered}
$$

where $z$ denotes the probability that $I$ chooses $d=1$. We use the following Lemma.

Lemma 3 When the award is confidential, at the solution we have: $\alpha_{1}^{1}, \alpha_{2}^{2}>\gamma>\alpha_{1}^{2}, \alpha_{2}^{1}$. 
Proof of Lemma 3. By taking the difference between $\alpha_{\sigma_{i}}^{d}$ and $\gamma$, we have $\alpha_{2}^{2}>\gamma>\alpha_{2}^{1}$, and $\alpha_{1}^{1}>\gamma>\alpha_{1}^{2}$ if $z \leq \frac{1}{(2-\nu)}$ and vice versa. Suppose that $z>\frac{1}{(2-\nu)}$ and therefore $\alpha_{2}^{2}, \alpha_{1}^{2}>\gamma>\alpha_{1}^{1}, \alpha_{2}^{1}$. Then (??) implies: $\nu\left(\alpha_{2}^{2}-\gamma\right)=(2-\nu)\left[\left(\gamma-\alpha_{1}^{2}\right)\right.$, which cannot hold since $\gamma<\alpha_{2}^{2}, \alpha_{1}^{2}$. Therefore it must be $z \leq \frac{1}{(2-\nu)}$.

In light of Lemma 3, condition (??) becomes

$$
\begin{aligned}
\nu\left(\alpha_{2}^{2}-\gamma\right)= & (2-\nu)\left(\alpha_{1}^{1}-\gamma\right), \\
& i . e, \\
\frac{\nu z}{\gamma+(1-\gamma)(1-z)}= & \frac{(2-\nu)(1-(2-\nu) z)}{\gamma+(2-\nu)(1-\gamma) z}
\end{aligned}
$$

Let $l(z) \equiv \frac{\nu z}{\gamma+(1-\gamma)(1-z)}$ and $r(z) \equiv \frac{(2-\nu)(1-(2-\nu) z)}{\gamma+(2-\nu)(1-\gamma) z}$ in the above expression, thus $l(z)-r(z)$ is the difference in the expected payoff of $I$ from choosing $d=2$ rather than $d=1$. Note that $l\left(z^{l s}\right)$ is increasing in $z$, with $l(z=0)=0$ and $l(z=1)=\frac{\nu}{\gamma}$, whilst $r(z)$ is decreasing in $z$, with $r(z=0)=\frac{(2-\nu)}{\gamma}>0$ and $r(z=1)=\frac{-(1-\nu)(2-\nu)}{\gamma+(2-\nu)(1-\gamma)}<0$. Thus $l(z)-r(z)$ is increasing in $z$ : the greater the probability $z$ that $I$ chooses $d=1$ the lower the posterior belief of $P_{1}$, upon observing $d=1$, that the arbitrator is competent . Furthermore, a solution to (14) with $z>0$ always exists and it is unique. ${ }^{13}$ At $z=\frac{1}{2}$ we have

$$
l\left(z=\frac{1}{2}\right)-r\left(z=\frac{1}{2}\right)=\frac{\nu \frac{1}{2}}{\gamma+(1-\gamma) \frac{1}{2}}-\frac{(2-\nu) \nu \frac{1}{2}}{\gamma+(2-\nu)(1-\gamma) \frac{1}{2}} \begin{cases}<0 & \text { for } \nu \neq 0,1 \\ =0 & \text { for } \nu=0,1\end{cases}
$$

which suggests that at $z=\frac{1}{2}, I$ strictly prefers to choose $d=1$ to $d=2$ for $\nu \neq 0,1$; whilst $z=\frac{1}{2}$ is an equilibrium for $\nu=0,1$.

Proof of Corollary 1. Suppose that also $P_{2}$ is long-lived and let $\beta_{\sigma_{2}}^{d}$ denote his posterior belief that the arbitrator is competent following a decision $d$, upon observing a signal $\sigma_{2}$ and using players' equilibrium strategies. Following the same procedure as for the case of asymmetric parties, the payoff of $C$ from choosing $d=\theta$ is given by

$$
\begin{aligned}
& V(d=1 \mid \theta=1)=(1-\nu) \alpha_{1}^{1}+\nu \beta_{1}^{1} \\
& V(d=2 \mid \theta=2)=(1-\nu) \beta_{2}^{2}+\nu \alpha_{2}^{2}
\end{aligned}
$$

\footnotetext{
${ }^{13}$ Since $r\left(z=\frac{1}{(2-\nu)}\right)=0$, at the solution we must have $z<\frac{1}{(2-\nu)}$.
} 
whilst the payoff of $I$ is

$$
\begin{aligned}
& V(d=1)=\frac{1}{2}\left[(2-\nu) \alpha_{1}^{1}+\nu \beta_{1}^{1}\right] \\
& V(d=2)=\frac{1}{2}\left[(2-\nu) \beta_{2}^{2}+\nu \alpha_{2}^{2}\right]
\end{aligned}
$$

Therefore, the mixed strategy condition becomes

$$
(2-\nu) \alpha_{1}^{1}+\nu \beta_{1}^{1}=(2-\nu) \beta_{2}^{2}+\nu \alpha_{2}^{2}
$$

leading to $\widehat{z}^{l l}=\frac{1}{2}$. A similar procedure shows that $\widehat{z}^{s s}=\frac{1}{2}$ when both $P_{1}$ and $P_{2}$ are shortlived.

Proof of Proposition 2 From Lemma 2, and from (5) and (6), the expected payoff of $C$ when he exerts effort is given by

$$
E V^{C}=\frac{1}{2}\left(\alpha_{1}^{1}+(1-\nu) \gamma+\nu \alpha_{2}^{2}\right)
$$

whilst the expected payoff of $C$ when he chooses $e^{C}=0$ from Lemma 2 and from (7) is given by (using the fact that $V(d=1)=V(d=2)$ )

$$
E V^{I}=\frac{1}{2}\left((2-\nu) \alpha_{1}^{1}+\nu \gamma\right)
$$

Taking the difference $E V^{C}-E V^{I}$ yields $\widehat{\Psi}^{l s}$ in expression (8). Differentiating $\widehat{\Psi}($.$) with$ respect to $\widehat{z}^{l s}$ we have $\frac{\partial \widehat{\Psi}(.)}{\partial \widehat{z}^{l s}}<0$ for $\widehat{z}^{l s}>\frac{1}{2}$.

Proof of Proposition 3. By repeating the same procedure as in Proof of Proposition 2and by using the Proof of Corollary 1, we obtain $\widehat{\Psi}^{s s}=0$ and

$$
\widehat{\Psi}^{l l}=\frac{1}{2}\left(\alpha_{1}^{1}-\gamma\right)+\frac{1}{2}\left(\beta_{2}^{2}-\gamma\right)
$$

where $\beta_{2}^{2}=\alpha_{1}^{1}$, since $z^{l l}=\frac{1}{2}$.

Proof of Proposition 4. Using Bayes rule we have

$$
\begin{gathered}
\gamma^{1}=\frac{\frac{1}{2} \gamma}{\frac{1}{2} \gamma+(1-\gamma) z} \\
\gamma^{2}=\frac{\frac{1}{2} \gamma}{\frac{1}{2} \gamma+(1-\gamma)(1-z)}
\end{gathered}
$$

where $\gamma^{1}>\gamma>\gamma^{2}$ for $z<1 / 2$ and vice versa. The mixed strategy condition now leads to

$$
\nu\left[\max \left\{\gamma^{2}, \alpha_{2}^{2}\right\}-\max \left\{\gamma^{1}, \alpha_{2}^{1}\right\}\right]=(2-\nu)\left[\max \left\{\gamma^{1}, \alpha_{1}^{1}\right\}-\max \left\{\gamma^{2}, \alpha_{1}^{2}\right\}\right]
$$


Taking the difference $\alpha_{\sigma_{i}}^{d}-\gamma^{d}$, using (13) and (15), it is easy to show that an equivalent version of Lemma 3 continues to hold. In particular, $\alpha_{\sigma_{1}}^{d=\sigma_{1}}>\gamma^{d}>\alpha_{\sigma_{1}}^{d \neq \sigma_{1}}$ for $\nu, z>0$. Condition (16), then becomes

$$
\nu\left[\alpha_{2}^{2}-\gamma^{1}\right]=(2-\nu)\left[\alpha_{1}^{1}-\gamma^{2}\right]
$$

which can be rewritten as

$$
\nu\left(\alpha_{2}^{2}-\gamma\right)-(2-\nu)\left(\alpha_{1}^{1}-\gamma\right)=\nu\left(\gamma^{1}-\gamma\right)+(2-\nu)\left(\gamma-\gamma^{2}\right)
$$

where

$$
\nu\left(\gamma^{1}-\gamma\right)+(2-\nu)\left(\gamma-\gamma^{2}\right)\left\{\begin{array}{lll}
\geq 0 & \text { if } & z \leq 0.5 \\
\leq 0 & \text { if } & z \geq 0.5
\end{array}\right.
$$

Let $\widetilde{z}^{l s}$ denote the solution in $z$ to (17) and suppose, by contradiction, that $\widetilde{z}^{l s} \leq 1 / 2$ for $\nu \neq 0,1$. Then from (17) and (18), the $L H S$ of (17) must be positive. Since the $L H S$ of (17) is increasing in $z$ and equal to zero at $z=\widehat{z}$ (from Proposition 1 and in particular condition 14) it follows that $\widetilde{z} \geq \widehat{z}$. Since $\widehat{z}>0.5$ (from Proposition 1), we have a contradiction. Therefore, $\widetilde{z}^{l s}>0.5$. This in turn implies from (18) that at $z=\widetilde{z}^{l s}$, the LHS of (17) must be negative. Using the fact that the LHS is increasing in $z$ and equal to zero for $z=\widehat{z}^{l s}$ it follows that $\widetilde{z}^{l s} \in\left(\frac{1}{2}, \widehat{z}^{l s}\right)$

Proof of Lemma 1. From Lemma 2, the expected payoff of $C$ when he chooses $e^{C}=1$ is given by

$$
E V^{C}=\frac{1}{2}\left[\alpha_{1}^{1}+(1-\nu) \gamma^{2}+\nu \alpha_{2}^{2}\right]
$$

whilst the expected payoff of $C$ when he chooses $e=0$ coincides with that of $I$, given by

$$
E V^{I}=\frac{1}{2}\left[(2-\nu) \alpha_{1}^{1}+\nu \gamma^{1}\right]
$$

Taking the difference between the two expected payoffs we have

$$
E V^{E}-E V^{I}=\frac{1}{2}\left[\nu\left(\alpha_{2}^{2}-\gamma^{1}\right)-(1-\nu)\left(\alpha_{1}^{1}-\gamma^{2}\right)\right]
$$

which in light of $\nu\left(\alpha_{2}^{2}-\gamma^{1}\right)=(2-\nu)\left(\alpha_{1}^{1}-\gamma^{2}\right)$ from (16), yields

$$
\widetilde{\Psi}^{l s}(\gamma, \nu)=\frac{1}{2}\left(\alpha_{1}^{1}\left(\widetilde{z}^{l s}(\gamma, \nu)\right)-\gamma^{2}\left(\widetilde{z}^{l s}(\gamma, \nu)\right)\right)
$$

Simple computations show that for $\widetilde{z}^{l s}()=.\widehat{z}^{l s}()=0.5,. \widetilde{\Psi}()=.\widehat{\Psi}($.$) . Since 0>\frac{\partial}{\partial \widetilde{z}^{l s}} \widetilde{\Psi}()>$. $\frac{\partial}{\partial \widetilde{z}^{l s}} \widehat{\Psi}($.$) , it follows that \widetilde{\Psi}()>.\widehat{\Psi}($.$) , for \widetilde{z}^{l s}()<.\widehat{z}^{l s}($. 
Proof of Proposition 5 It follows from $F_{\chi \Psi}() \geq$.0 , implying $F_{\chi}(\widetilde{\Psi}(),. \chi) \leq F_{\chi}(\widehat{\Psi}, \chi)$.

Proof of Proposition 7. With a slight abuse of notation, let us denote by $\widehat{U}_{k}^{A}$ and $\widetilde{U}_{k}^{A}$ the expected payoff of the parties in case $k$ under arbitration, respectively when there is a confidentiality provision and when there is not. Following the same procedure as in Section 3, we have $\widehat{U}_{A}^{l l}>\widehat{U}_{A}^{l s}>\widehat{U}_{A}^{s s}$. (ii). Comparing $\bar{U}$ with $\max \left\{\widetilde{U}_{A}^{k}, \widehat{U}_{A}^{k}\right\}$, noting that $\bar{U}(r)$ is increasing in $r$, with $\bar{U}(r) \geq \widetilde{U}_{s s}^{A}=\frac{1}{2}(1+F(\chi) \gamma)$, for all $r$, and $\left.\bar{U}(r=1)>\widetilde{U}_{l l}^{A}>\bar{U} r=0\right)$, the result follows.

\section{References}

[1] Anderlini, L., Felli, L. and A. Postlewaite, (2006): 'Should Courts Always Enforce What Contracting Parties Write?,' Working Papers London School of Economics.

[2] Bingham, L.B., (1998), 'On repeat players, adhesive contracts, and the use of statistics in judicial review of employment arbitration awards,' McGeorge Law Review, 223.

[3] Bloom, D. and C. Cavanah (1986), 'An Analysis of the Selection of Arbitrators,' American Economic Review, 76: 408-422.

[4] Bourjade, S. and B. Jullien (2005), 'Expertise and Bias in Decision Making, 2005, Working Papers, IDEI, University of Toulouse.

[5] Chakravarty, S. and M. Makris (2005), 'Resolving Contractual Disputes', CMPO Working paper, $1 \%$.

[6] Che, Y.K (1995), 'Revolving Doors and the Optimal Tolerance for Agency Collusion, ' RAND Journal of Economics, 26: 378-397.

[7] Dixit, A. (2003): 'Arbitration and Information'. Working Paper, Princeton University.

[8] Drahozal, C. F. (2001), 'Unfair Arbitration Clauses', University of Illinois Law Review, 695.

[9] Fitz, A. (1999), 'The debate over the mandatory arbitration in employment disputes'. Dispute Resolution Journal, 54. 
[10] Heyes, A. (2003), 'Expert Advice and Regulatory Complexity,' Journal of Regulatory Economics, 24: 119-133.

[11] Iossa, E. and G. Palumbo (2005), 'Information Provision and Monitoring of the Decisionmaker in the Presence of an Appeal Process,' Working Papers Brunel University.

[12] Leaver, C. (2004), 'Bureaucratic Minimal Squawk Behavior: Theory and Evidence from Regulatory Agencies'. Working Papers, University of Oxford.

[13] Levy, G. (2004), 'Anti-herding and Strategic Consultation', European Economic Review, 48: 503-525.

[14] Levy, G. (2005), 'Careerist Judges,' RAND Journal of Economics, 36: 275-297.

[15] Miceli, T. and Coşgel, M. (1994), 'Reputation and Judicial Decision-Making,' Journal of Economic Behavior and Organization, 23: 31-51.

[16] Ottaviani, M. and Soerensen, P. (2006), 'Reputational Cheap Talks', RAND Journal of Economics, 37: 155-175.

[17] Salant, D. (1995), 'Behind the Revolving Door: a New View of Public Utility Regulation, RAND Journal of Economics, 26: 362-77..

[18] Shavell (1995), 'Alternative Dispute Resolution: an Economic Analysis,'. Journal of Legal Studies, 24: 1-24.

[19] Schweizer, U. (1989), "Litigation and Settlement under Two-Sided Incomplete Information,' Review of Economic Studies, 56: 163-177. 